\title{
Translation and translanguaging pedagogies in intercomprehension and multilingual teaching
}

\section{Pédagogies de traduction et de translanguaging dans le contexte de l'intercompréhension et de l'enseignement plurilingue}

\author{
Cedric Joseph Oliva \\ coliva1@bryant.edu \\ BRYANT UNIVERSITY \\ Clorinda Donato \\ clorinda.donato@csulb.edu \\ CALIfornia State University, Long BeACH \\ Francesca Ricciardelli \\ francesca.ricciardelli@upf.edu \\ UNIVERSITAT POMPEU FABRA
}

\begin{abstract}
Since 2007, California State University, Long Beach has developed and offered courses that highlight students' pre-existing linguistic repertoires in English and in the Romance languages. These courses are unique in that they build upon a multilingual base for the acquisition of new languages through the method of intercomprehension. As an approach that moves among languages, Intercomprehension places learners in conditions that are conducive to translanguaging and translation. This paper discusses the role of translation and translanguaging in Intercomprehension as a pedagogical approach in these courses. Since our students are constantly moving between English and one or more Romance language(s), they actively bring the other Romance languages they are learning into the translingual repertoire they already practice through the multilingual learning strategies deployed in intercomprehension.
\end{abstract}

Key words: intercomprehension, plurilingualism, multilingualism, pedagogies, translanguaging, translingual practices 


\begin{abstract}
Résumé
Depuis 2007, California State University, Long Beach développe et offre des cours qui mettent en avant le répertoire linguistique préexistant des étudiants en anglais et en langues romanes. Ces cours sont uniques, car ils s'appuient sur un répertoire multilingue pour permettre l'acquisition de nouvelles langues à travers la méthode d'intercompréhension. L'intercompréhension, approche transcendant les barrières entre les langues, offre aux apprenants un contexte propice au translanguaging et à la traduction. Cet article discute du rôle de la traduction et du translanguaging dans l'intercompréhension. Étant donné que nos étudiants naviguent constamment entre l'anglais et une (ou, des) langue(s) romanes(s), ils font ainsi entrer de manière active les tierces langues romanes en cours d'apprentissage dans le répertoire translangagier qu'ils utilisent déjà par le biais des stratégies intercompréhensives.
\end{abstract}

Mots-clés : intercompréhension, plurilinguisme, multilinguisme, pédagogies, translanguaging, pratiques translangagières

\title{
Introduction and background
}

The presence of Spanish in any number of urban landscapes across the United States bespeaks a unique set of geo-historical contexts characterized by conquest, displacement, return, migration, and geographical proximities, such as those that prevail in borderlands - that is, the current highly fraught situation on the Mexican-U.S. border. The confluence of so many factors in the mapping of Spanish and its vitality today in the United States constitutes a veritable language geography whose contours have become increasingly visible and mainstream, indeed, the condition of new language geographies that Simon (2012) has spoken about in Cities in translation: Intersections of language and memory to describe "topographies of language and their power to mark the urban landscape" (p. xix). Throughout the United States and to some extent Canada as well, Spanish has become an element of a new language geography that is changing the linguistic landscape of every sector of life and culture. Certain areas of the United States, such as some sectors of California, Florida, and New York, can be considered both Spanish-speaking and English-speaking in equal measure. The ways in which new language geographies generate new language practices has been at the forefront of García's research. Her observations of children in multilingual contexts revealed their unified language repertoire and their natural movement within it. Designated as translanguaging, echoing the work of Williams (1994), this movement among languages in one's repertoire has established a new paradigm for the study of language acquisition, which has engendered a highly nuanced terrain for innovative research as the contributions to this volume demonstrate. 
In this article, we relate our work on the use of translation and translanguaging pedagogies in language acquisition among multi- and plurilingual learners (García, 2007). At California State University, Long Beach (CSULB) in Southern California, a geographical region where growing numbers of students in educational institutions at every level are emerging English-Spanish bilinguals, we have developed teaching pedagogies that work in tandem with a student's entire linguistic repertoire to teach languages that are typologically similar to Spanish, in this case, French and Italian. This article focuses on the courses we have developed - "French and Italian for speakers of English and Spanish," and "Intercomprehension among Romance languages" - as well as the materials we have used, and those we have created for teaching in multiple languages. Data from the teaching of the intercomprehension course at St. Lawrence University in upstate New York is also included.

\section{The emergence of new perspectives and opportunities for a growing audience}

As keynote speaker at the 2011 Kentucky Foreign Language Conference, Mary Louise Pratt delivered the lecture "Globalization as linguistic force field," in which she offered several examples from hip-hop and rap music where the multiple languages of the artists formed translanguaging texts widely embraced across North America (Pratt, 2011, p. 6). Pratt referenced the dissemination of this urban, multilingual, and multicultural music as the bellwether for global music. Indeed, what was a trend in 2011 has become a solidly entrenched new paradigm for music in 2019. The bilingual phenomenon has continued to evolve in urban music to such a point that the English and Spanish musical markets are destined to become unified, as increasing numbers of English-language singers produce music in Spanish for the burgeoning Spanish-language market, and Spanish rap artists and rhythms, such as Reggaeton and Latin rap continue to garner audiences and emulators worldwide. ${ }^{1}$ Some fifty years following the British invasion in music, we witness now the Spanish-English invasion, where a translanguaging rap version of "I like it like that" (Pabon \& Rodriguez, 1967) reached number one in 2018. Its signature feature resides in the ability of the three rappers, Cardi B, J. Balvin, and Bad Bunny, to rap seamlessly in SpanishEnglish, a talent that many artists, including Justin Bieber and Rihanna, seek to replicate in their own work. The success of bilingual music over the last few years demonstrates the power of new language geographies to create and disseminate cultural products that reflect the reality of an expanded linguistic repertoire for greater numbers of citizens. News outlets had been reporting

\footnotetext{
${ }^{1}$ We note that Reggaeton music originated in the late 1990s in Puerto Rico and the Caribbean. It is characterized by rapping and singing in Spanish.
} 
on the demographic shifts for some time. In April 2014, CNN sparked media attention after featuring an article entitled "California's Hispanic milestone" (Ruben, 2014) announcing a major demographic shift in the state of California, where the Hispanic population would soon become predominant. A year later, the New York Post noted that the United States accounted a higher number of Spanish speakers than Spain (Perez, 2015). Indeed, the Post's article reported the results from a study conducted by the Instituto Cervantes that estimated an all-inclusive Spanish speaking population of 56.2 million people (including native, heritage, and academic speakers), thus placing the United States as the second most populated Spanish speaking country in the world after Mexico (121 million speakers) but more than Colombia (48 million) and Spain (46 million).

Yet, the socio-linguistic shift which is palpable on the streets and on university campuses is largely ignored in educational institutions, which still consider as problematic the use of any language other than English as the educational point of reference. Particularly in the language classroom, bilingual and multilingual students are asked to forget that their linguistic repertoire includes languages other than English. In this context, Hispanic-serving institutions such as CSULB - which reports a student body that is over $40 \%$ Hispanic (California State University, 2019) - seek ways to better integrate students with bilingual and bicultural profiles by presenting them with teaching and learning strategies that allow them to work from these linguistic profiles as points of strength. Thanks to the favourable demographic conjuncture (Oliva \& Donato, 2015) in the university's Department of Romance, German, Russian Languages and Literature, the support of the Services Culturels du Consulat de France à Los Angeles, and a three-year National Endowment for Humanities grant, we were able to address the growing numbers of Spanish-speaking students in pedagogically inclusive ways in our French and Italian classes. The most immediate objective was to offer bilingual students introductory- and intermediate-level curricula specifically tailored for speakers of English and Spanish. In this endeavour, French and Italian courses were adapted to the students' existing linguistic knowledge as well as their metacognitive (translingual) awareness as experienced bilinguals. While we will discuss both points in the next two sections, in this section, we would like to survey the considerable evolution of research in the United States and internationally that has shed light on multilingual teaching for multilingual students. Acknowledging the existence of learners with new linguistic and cultural backgrounds was a turning point in the advancement of research and the development of educational and pedagogical projects (Kramsch, 2009; Kramsch \& Zhang, 2018).

In many instances, promotion of such projects remains complicated to implement in American institutions as Gramling and Warner described in 2015: 
"[a]s a field, critical multilingualism studies has to rely on intergroup, interlingual, transnational, and international research communities... Participants in these communities often operate in knowledge domains that are 'structurally obscured in Anglocentric institutions'" (p. 3). In the case of L3 education in Europe, Portolés and Martí (2017, p. 64) note that multilingual education still abides by monolingual principles. Lemke (2002) remarks that political and ideological pressures render the development of multilingual education more complicated than it needs to be. Furthermore, pushed by ideological, sociological, or political norms, research and instruction alike have developed a tendency to regard multilingualism as a series of parallel monolingualisms (Bailey, 2007; Grosjean, 2010) which fit nationalistic language archetypes. However, such archetypes contradict the actual linguistic reality of multilingual speakers who, even when influenced by ideological, social, or political messages about monolingualism, nevertheless experience their language repertoire as whole, no matter how many languages exist within it.

Language policies that are unfriendly to multilingualism weigh heavily upon many potentially multilingual teaching contexts throughout the world. Such cases have been reported in the context of African bilingualism (Makoni \& Mashiri, 2007) or Corsican bilingualism (Jaffe, 1999). In Ideologies in action, Jaffe describes advances made in the inclusion of Corsican in all of its forms as a viable and teachable language. Indeed, Corsican researchers and policy-makers advocated for successfully moving away from the commonly accepted diglossic perspective and situation in place in the 80s, which offered an unbalanced power dynamic between French (High) and Corsican (Low) and nurtured, walled-off, social, and educational norms. The developing ideologies lead to the acceptance of polynomic status and approaches to language variations on the island. This marks the emergence of an important shift in policies where "[t]he concept of polynomy did not advocate a power reversal, but rather, a redefinition of what power was" (p. 186) leading to the acceptance of all variations of Corsican. However, as Jaffe observes, recognition of how bilinguals draw from their entire linguistic repertoire when speaking and learning had not yet entered mainstream thinking about language ideologies. Therefore, these changes

revealed the difficulty of applying the idea of polynomic language against a backdrop of deeply rooted ideas about linguistic legitimacy in the face of practical requirements for teaching. ... Nor did it seem that tolerance of diversity was extended to mixed forms, whether they were mixtures of Corsican and French or mixtures of Corsican dialects. (Jaffe, 1999, p. 187)

The recent efforts of the Belgian school system in offering more diversified and more accessible opportunities for bilingual education is a salient example of actions taken to offer more appropriate policies which place "[l]'édu- 
cation bilingue au coeur des politiques linguistiques" (Babault, 2015, p. 38). In fact, research interest is burgeoning, marked by the growing number of journals dedicated to the topic, such as the creation of Critical Multilingualism Studies in 2012, followed by Translation and Translanguaging in Multilingual Contexts in 2015, not to mention the large number of articles and monographs (Burck, 2005; Kramsch, 2009; Kramsch \& Zhang, 2018). In fact, as early as 2005, the pedagogical value and potential of codeswitching was described by Lin and Martin (2005). In the context of heritage language learners, Cummins (2005) argues the necessity of adapting bilingual educational strategies to take into account the fully functioning cross-linguistic transfers that exist between two languages. All such contributions lend social and educational validity to codeswitching.

In their descriptions of bilingual classrooms, García (2007, 2009) and Creese and Blackledge (2010) evidence the value of the pre-acquired linguistic repertoire of bilinguals and their inherent translanguaging as a linguistic "toolkit" that equips bilingual learners for educational success. Attempting to eliminate the natural tendency of bilinguals to translanguage, and to impose upon them instead a monolingual identity destroys their inherent capacity to make connections between all of the languages they know and all of the languages they are learning. Traditionally, in the context of modern language learners in the United States, monolingual neophyte learners of French were discouraged from using their knowledge of English to develop translanguaging techniques and skills between English and French. The well-anchored, persistent, and pervasive (still today) preference for "target-language-(only)" approaches removes all languages except the target language from acquisition strategies. In the same manner, bilingual (English-Spanish) neophyte learners of French were not encouraged to use their knowledge of English, not to mention Spanish, or their predisposition to use interlanguaging pathways between English, Spanish, "Spanglish" and French as they acquired the language. Most commonly used textbooks offer no tools or approaches that elicit such connections.

In the case of L3+ acquisition, the next section sheds light on the advantages that flexible and customized approaches offer in the acquisition process. It shows how the use of all pre-acquired languages, as well as connections existing among typologically similar languages, become an asset for learning. The journal Translation and Translanguaging in Multilingual Contexts presents translanguaging and translation, not only as communicative phenomena but also as "advanced pedagogies" that embrace the co-existence of multiple languages in the implementation and creation of pedagogical approaches. The courses, teaching pedagogies, and materials produced in this article reflect these principles. 
OLIVA ET AL. Pedagogies in intercomprehension/l'enseignement plurilingue

\section{L'enseignement du français, de l'italien et du portugais pour locuteurs anglo-hispanophones : de nouveaux buts pour les programmes de langues}

Pour nous adapter au contexte décrit dans la précédente section, nous avons créé une offre pédagogique intégralement repensée et restructurée qui tient compte du nombre toujours grandissant d'étudiants bilingues et surtout anglohispanophones (Oliva, 2014-2016; Donato et Oliva, 2016; Oliva et Donato, 2015). Depuis la rentrée de l'automne 2007, nous avons créé un cours expérimental de français de premier semestre débutant (100A) spécialement conçu pour ces étudiants bilingues. Au printemps 2008, s'en suivait la création du cours de premier semestre débutant (100A) d'italien. Le succès de ces cours niveau débutant nous a menés au développement d'un programme d'étude complet de trois semestres (débutant et intermédiaire) en français puis en italien. Dès le semestre du printemps 2009, nous offrions déjà tous les niveaux (100A, 100B et 200) dans les deux langues. Traditionnellement, nos programmes débutant et intermédiaire s'effectuent en quatre semestres d'enseignement. Mais ces programmes répondant aux besoins des étudiants bilingues, nous ont permis de prendre en considération les capacités particulières de ces étudiants et de créer un programme intensif et accéléré permettant de couvrir le contenu de quatre semestres en trois semestres (Fig. 1).

\begin{tabular}{|c|c|c|c|c|c|}
\hline & $\begin{array}{l}\text { Premier } \\
\text { semestre }\end{array}$ & $\begin{array}{c}\text { Second } \\
\text { semestre }\end{array}$ & $\begin{array}{l}\text { Troisième } \\
\text { semestre }\end{array}$ & $\begin{array}{l}\text { Quatrième } \\
\text { semestre }\end{array}$ & \\
\hline $\begin{array}{l}\text { Cursus } \\
\text { traditionnel }\end{array}$ & $101 \mathrm{~A}$ & 101B & $201 \mathrm{~A}$ & 201B & Cours \\
\hline $\begin{array}{c}\text { Cursus pour } \\
\text { « anglo- } \\
\text { hispanophones» }\end{array}$ & $100 A$ & $100 \mathrm{~B}$ & $\begin{array}{c}200 \\
\text { Cours intensif, } \\
\text { hybride, intégralementment } \\
\text { renversé ou « flipped " }\end{array}$ & & $\begin{array}{l}3^{e} \text { année } \\
\text { (minor) }\end{array}$ \\
\hline
\end{tabular}

FIGURE 1

Comparaison du cursus traditionnel et du cursus dit « anglo-hispanophone » aux niveaux débutant (100/101) et intermédiaire (200/201)

Du fait que ces cours soient basés sur une approche intercompréhensive (Fig. 5), il existe une certaine marge de manœuvre concernant les compétences prérequises nécessaires pour pouvoir les suivre. La plupart de nos étudiants ont une compétence avancée en espagnol (équivalent 3ème année universitaire), qu'elle soit native, d'héritage académique, ou une combinaison des deux. Dans un esprit de fluidité pédagogique, nous voulions que les étudiants ayant une compétence linguistique dans une langue proche autre que l'espagnol puissent 
également suivre ces cours. Par exemple, nous avons reçu dans les cours de français et d'italien des étudiants luso-anglophones et roumano-anglophones, dans le cours d'italien des franco-anglophones et dans le cours de français des italo-anglophones. Et aussi quelques rares exceptions comme une étudiante germano-anglophone et une étudiante monolingue suivant un cursus de baccalauréat en linguistique. Oliva a mené un questionnaire détaillé d'entrée et de sortie de cours durant la période de l'automne 2014 au printemps 2016 (Oliva, 2014-2016). Ce questionnaire ${ }^{2}$ a permis notamment de recueillir les statistiques linguistiques des 260 étudiants ayant suivi les cours de langue de niveau débutant et intermédiaire pour « anglo-hispanophones» durant cette période (Fig. 2).

Bien que les composantes intercompréhensives (Fig. 5) permettent un certain dégré de fluidité linguistique dans ces cours, ils restent, in fine, des cours de langues avec une focalisation sur le développement de la compétence de communication en langue cible et suivent les lignes directrices de compétences langagières telles que celles déterminées par l'ACTFL (s.d.). Pour ce qui est des transparences lexicales, nous invitons les apprenants à développer des compétences de lecture avancées en langue cible dès le début de l'enseignement. L'apprentissage avancé de la lecture a une incidence positive sur les trois autres compétences (Escudé et Janin, 2010). Ce processus se développe autour d'apprentissage permettant de repérer les mots transparents et de négocier les différents niveaux de transparence en déduisant les faux-amis grâce au contexte. Il s'appuie sur les connaissances provenant du répertoire des étudiants, c'est-à-dire les connaissances, en général, de l'anglais et de l'espagnol.

L'étude du comportement systématique et des stratégies automatiquement mises en œuvre par les apprenants bilingues nous a permis de réaliser qu'une approche uniquement basée sur un «étayage pédagogique» ne pouvait leur convenir. Par exemple, dans le cadre d'un cours traditionnel traitant des verbes pronominaux en français, le modelage progressif de la forme pronominale inclut souvent : une révision des terminaisons verbales au présent et de la conjugaison, un modelage «me/te $(x+1$ introductif $)$, une explicitation du concept de réflexivité, un modelage des autres formes «me/te/se/nous/vous/se » $(x+1$ suite), des exercices de modelage, des exercices d'utilisation, et finalement des exercices communicatifs et/ou des activités de taches. Nos étudiants bilingues démontrent, et ce dès les premiers instants, une compréhension précise du $\mathrm{x}+1$

\footnotetext{
${ }^{2}$ Oliva a réalisé un questionnaire d'entrée et de sortie des cours durant quatre semestres (de l'automne 2014 au printemps 2016). Le questionnaire a également permis de récolter des valeurs de connaissances lexicales, grammaticales et métalinguistiques qui conduiront à d'autres études de résultats. 506 étudiants ont été interrogés dont 260 dans les cours spécialisés (français/italien pour locuteurs d'anglais et d'espagnol) et 246 dans les cours traditionnels.
} 


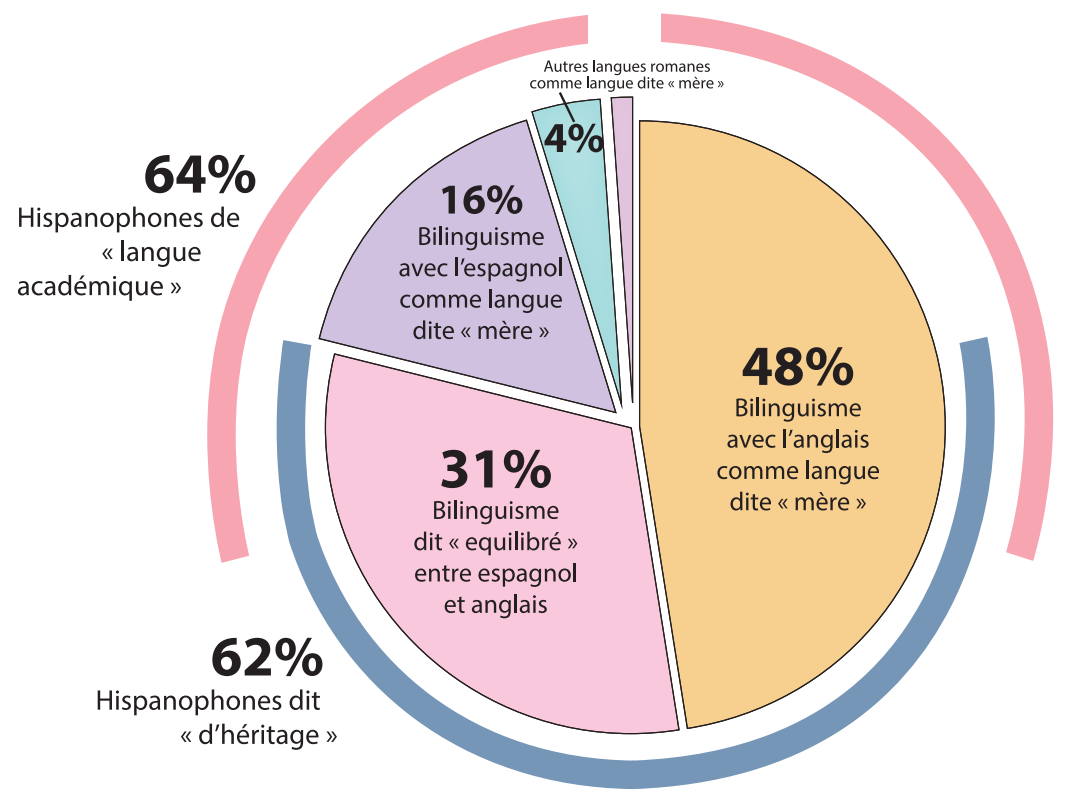

Figure 2

Statistiques des connaissances linguistiques des 260 étudiants ayant participés aux cours de français ou d'italien pour anglo-hispanophones de l'automne 2014 au printemps 2016. (Oliva, 2014-2016, données du questionnaire)

et du concept de réflexivité qui est en général déjà acquis et de ses modes et conditions d'utilisation. Les questions et difficultés de nos étudiants se portent primordialement sur les points de divergences tels que la forme « vous vous » pour les locuteurs hispanophones familiers de « ustedes se » au lieu de «vosotros os. » En effet, dans ce cas, l'approche traditionnelle, n'offre que peu de moyens et d'outils pour permettre aux apprenants de fructifier leur bilinguisme préexistant; et donc, de se servir de leur répertoire linguistique respectif pour le connecter, par un translanguaging qu'ils appliquent déjà entre l'anglais et l'espagnol, à la langue en cours d'apprentissage.

Ces conditions d'apprentissage traditionnelles revenaient donc à faire avancer nos étudiants sur le difficile chemin de l'acquisition d'une langue nouvelle tout en leur mettant des œillères. C'est ainsi que dès la mise en place de ces cours en 2007, nous avons réalisé que le matériel venant de l'approche pédagogique standardisée que nous utilisions pour ces cours n'était pas suffisamment adapté. En effet, la plupart du matériel didactique disponible offrait sur une approche monolingue, externalisée et cloisonnée qui, pour l'apprentissage d'une 
nouvelle langue, jetait donc une ombre sur le répertoire linguistique préétabli des étudiants (Fig. 3). L'espagnol en était comme effacé, mis dans une prison linguistique, et l'anglais normatif ne pouvait servir que comme référent externe propice aux discussions sur les points grammaticaux ou culturels. Le français en cours d'acquisition, l'interlangue parfois même appelé pidgin, bien souvent considéré comme une version sous-classée et dissociée de la langue comme norme politico-idéologique, y demeurait quasi-perpétuellement en devenir (au niveau qualitatif) de cet élusif niveau standard du locuteur natif.

\section{« Répertoire » cloisonné et normatif de l'apprenant}
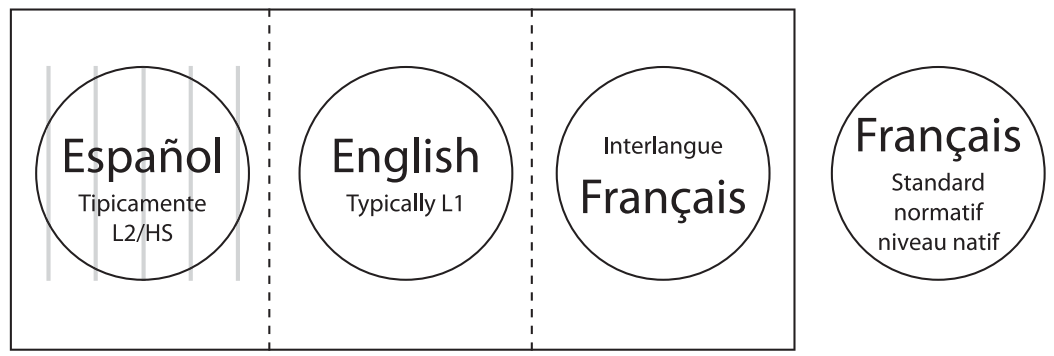

\section{FIGURE 3}

Perception traditionnelle, cloisonnée et normative du répertoire de l'apprenant

(Oliva, 2016)

Il est suggéré que, dans le cadre de l'enseignement des langues proches, une approche « consciente » de la grammaire est préférable (Donato et Oliva, 2014; Carvalho, Freire et Da Silva, 2016) par rapport à celle inductive provenant du scaffolding de l'approche communicative. Le concept de conscience et de noticing langagier, comme on le retrouve chez Schmidt et al. (2010), préconise que les processus d'acquisition conscients sont, sinon nécessaires, fortement facilitateurs pour l'acquisition d'une langue. Dans le cas de langues proches, il faut inculquer à l'apprenant comment négocier des différences infimes qui existent entre langues romanes (Carvalho, Freire et Da Silva, 2016). Même si ces dernières ne forment pas nécessairement des obstacles à la communication, elles provoquent bien souvent des erreurs (cf. exemple du « à » prépositionnel en Tableau 1) qui doivent cependant être traitées dans le processus d'apprentissage (Stevens, 1990).

Pour l'apprentissage de l'utilisation du futur périphrastique, aussi dit «futur proche » (Tableau 1), bien que la structure anglaise soit remarquablement proche, elle n'en reste pas moins opaque pour un œil non-averti. Or, les apprenants anglo-hispanophones vont immédiatement, avec ou sans l'aide de l'instructeur, trouver une aide dans la structure transparente qu'ils connaissent en 
OLIVA ET AL. Pedagogies in intercomprehension/l'enseignement plurilingue

TABLE 1

Le futur périphrastique français pour apprenants anglo-hispanophones

\begin{tabular}{l|l}
\hline \hline Espagnol & (Tú) Vas a estudiar en Canadá. \\
Français & Tu vas étudier au Canada. \\
Anglais & You are going to study in Canada. \\
\hline \hline
\end{tabular}

espagnol. Si les étudiants n'élicitent pas cette proximité par eux-mêmes, l'instructeur met en œuvre des stratégies intercompréhensives qui les portent à la découvrir et la comprendre par leur propre efforts grâce au support de l'itinéraire multilingue. En effet, les manuels de texte (Fig. 5) que nous avons développés dans le cadre de nos cours de français et d'italien pour locuteurs anglo-hispanophones, intitulés Juntos : Italian for speakers of English and Spanish (Donato, Oliva, Zappador-Guerra et Romero, 2014) et Juntos : French for speakers of English and Spanish (Oliva, Donato, Gomez et Romero, 2015) offrent une approche basée sur les principes de l'intercompréhension (Figs. 5 et 7) favorable au translanguaging.

Bien que français et espagnol soient, en termes d'intercompréhension, des langues proches et «transparentes, » elles comportent dans l'exemple du future périphrastique une légère différence syntaxique en ce que l'espagnol requiert l'utilisation du «à » prépositionnel. La nécessité de celui-ci explique bon nombre d'erreurs produites par transfert (que d'aucuns nommeraient « transfert négatif ») de l'espagnol vers le français comme cela pourrait être le cas dans l'exemple suivant : «Tu vas à étudier au Canada. »

Plutôt que de qualifier ce transfert de «négatif » et tenter de l'éviter intégralement, notre perspective est de profiter de ce transfert translinguistique par le biais de language noticing. En termes concrets, nous validons le bon sens linguistique dont fait preuve l'apprenant en trouvant dans ses ressources préacquises un mode de production dans la langue cible et nous reconnaissons l'effort productif de «Tu vas à étudier au Canada » qui démontre clairement que la structure générale du futur périphrastique est déjà acquise. Nous apportons cependant, avec une pédagogie que l'on pourrait nommer «pédagogie de (reconnaissance de) la divergence» (Oliva, 2011), un travail en language noticing. Ce travail peut s'effectuer par la correction de phrases erronées (Fig. 4) ou au travers d'exercices de reconnaissance structurelle multilingue de type intercompréhensif (Fig. 5). Le résultat reste similaire du fait que les étudiants apprennent à distinguer les structures grammaticales dissociées entre les deux (ou plus de deux) langues, reconnaissent et intègrent l'élément erronée ou nonnécessaire, et produisent donc des formes valides et dépourvues de transfert dit «négatif» dans la langue cible. 


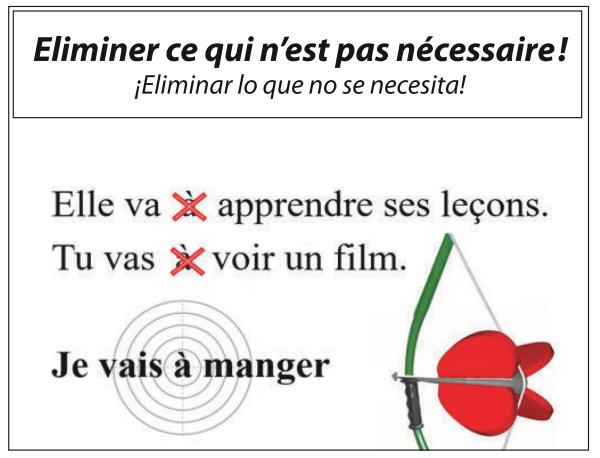

\section{FIGURE 4}

Pédagogie de la divergence (Oliva, 2011)

Dans un esprit semblable à celui de nos travaux, le rapport sur les politiques linguistiques du Conseil de l'Europe qui se focalise sur le cas de l'apprentissage de l'anglais langue seconde ou tierce soutient que le but des programmes de langues devrait être de favoriser le développement de compétences intégratives et multiples (plurilingues, interculturelles, etc.) (Seidlhofer, 2003). De ce fait, la poursuite d'une compétence de niveau de locuteur natif dans la langue cible ne devrait pas être la focalisation centrale des programmes de langues étrangères.

Les approches ayant pour unique but l'apprentissage niveau natif dans la langue cible en cloisonnement, porteront à apprendre la langue française avec le moins d'inférences possible de l'anglais, et en n'admettant bien évidemment pas que l'espagnol puisse jouer un rôle dans l'apprentissage (Fig. 3). Or, ces inférences font partie des processus d'apprentissage d'une langue tierce en translanguaging qui permet de faire rentrer la nouvelle langue dans le cercle de multicompétence (Cook, 1992) des apprenants. En effet, le répertoire linguistique ne peut être perçu comme une série de contenants indépendants et hermétiques, ou «leak proof» (Makoni et Mashiri, 2007) et pour que les apprenants prospèrent dans leur translanguaging, ils doivent faire preuve de «critical (metacognitive/linguistic) awareness » envers leurs pratiques langagières (Canagarajah, 2011; Donato et Oliva, 2016; García, 2007, 2011; Oliva et Donato, 2015; Seidlhofer, 2003).

Comme cela est exposé dans les figures 3 et 4, Juntos : French for speakers of English and Spanish « [p]resents multilingual examples that engage learners in exploring, deducing, and hypothesizing "about the concordances and divergences between target, reference and auxiliary languages while essentially performing exercises in the target language" » (Carvalho et Child, 2018, p. 423). Nous nous efforçons de réduire l'aspect négatif des labels apposés aux langues 


\section{Leçon 13}

\section{Le Future Périphrastique}

\section{Intercomprehension section}

1.1 Identify the languages:

Answer key:

PT, SP, FR:

IT: CO SC EN

(Eu) Vou estudar na universidage.

(Yo) Voy a hablar en francés.

Je vais regarder un film ce soir.

(Io) Sto per visitare Roma.

(Eiu) Aghju da parlà cù ellu.

(Iu) Vaiu a manciari una carota.

I am going to drink some water.

\section{$[\ldots]$}

1.3 Following the translation given for the Sicilian example "Vaiu a manciari", can you complete the rest of the chart in Spanish?

\begin{tabular}{|l|l|}
\hline (Tu) Vais estudar. & \\
\hline Elle va regarder. & \\
\hline (lu) Vaiu a manciari. & (Yo) Voy a comer. \\
\hline He is going to drink. & \\
\hline
\end{tabular}

Answer key:

\begin{tabular}{|l|}
\hline (Tú) Vas a estudiar. \\
\hline (Ella) Va a ver. \\
\hline (Yo) Voy a comer. \\
\hline (EI) Va a beber. \\
\hline
\end{tabular}

1.4 Observe the verbal structures of the Spanish sentences compared to the three other Romance languages in the chart. Spanish and Sicilian share a common feature not shared by French and Portuguese. What is it?

Answer key: The preposition "a" must be used between the auxiliary verb "to go" and the main action verb in Spanish and Sicilian. The preposition is not used in French and in Portuguese.

\section{Intergrammatical section}

2.1 Given your observations and the example below, can you complete the rest of the chart in French?

\begin{tabular}{|l|l|}
\hline (Tú) Vas a estudiar. & \\
\hline (Ella) Va a ver. & Elle va è regarder. \\
\hline (Yo) Voy a comer. & \\
\hline (El) Va a beber. & \\
\hline (Ellas) Van a cantar. & \\
\hline (Nos) Vamos a leer. & \\
\hline (Ellos) Van a hablar. & \\
\hline
\end{tabular}

\begin{tabular}{|l|}
\multicolumn{1}{c|}{ Answer key. } \\
\hline Tu vas è étudier. \\
\hline Elle va à regarder. \\
\hline Je vais à manger. \\
\hline Il va à boire. \\
\hline Elles vonnt è chanter. \\
\hline Nous allons è lire. \\
\hline Ils vonnt è parler. \\
\hline
\end{tabular}

\section{FiguRE 5}

Pédagogie de noticing intercompréhensif dans l'enseignement du français aux anglo-hispanones dans Juntos : French for Speakers of English and Spanish (Oliva, Donato, Gomez et Romero, 2015) 
Évolution du repertoire inclusif non-normatif de l'apprenant

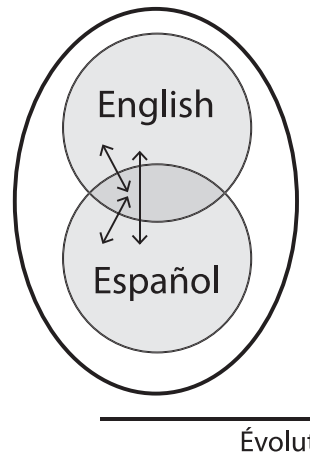

a.
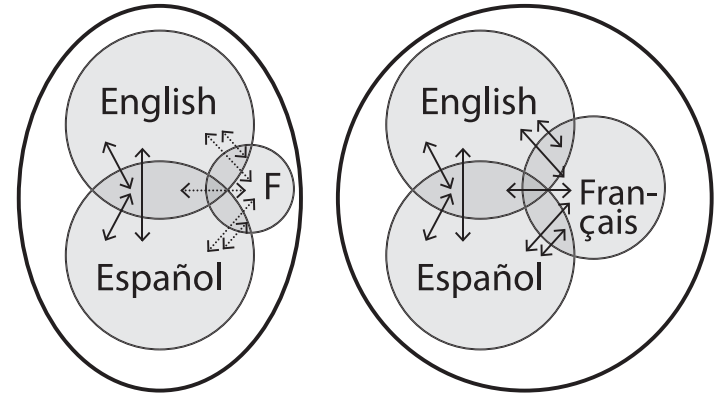

Évolution chronologique au fil de l'apprentissage

b.

c.

\section{Figure 6}

Perception inclusive et non-normative du répertoire de l'apprenant (Oliva, 2016)

(français cible/niveau natif, français interlangue, espagnol d'héritage, anglais d'éducation/natif, etc.) que l'on trouve dans un contexte traditionnel. Pour ce faire, nous proposons de prendre en considération la connaissance pré-acquise comme un ensemble (le répertoire linguistique de départ-Fig. 6a) quelles que soient leurs divergences d'origine (Fig. 2). Nous proposons de désacraliser la position anxiogène de «natif » de l'enseignant envers les apprenants en les exposant par pointillisme aux « langues auxiliaires » que ni apprenants, ni enseignants ne connaissent forcément mais que tous peuvent utiliser comme ressources permettant d'établir des connections translangagières par le fait qu'il s'agisse de langues proches. Cet enseignement se rapproche de certains des buts émis par le rapport de De Pietro et Rispail (2014), qui préconise d'inclure la didactique du français dans une didactique de la pluralité linguistique.

S'inspirant du fonctionnement de l'ensemble du répertoire linguistique de nos apprenants, ces étudiants sont habitués au «translinguisme » et sont donc équipés des outils nécessaires pour «naviguer » entre plusieurs langues (Fig. 6a). Nous avons pris en considération le fait que nos apprenants sont en général bilingues à différents niveaux (asymétriques, équilibrés, différés, dits «d'héritages, » académiques, locuteurs d'une autre langue romane, etc.) comme démontré par les résultats du questionnaire (Fig. 2; Oliva, 2014-2016) et c'est pour cette raison que le matériel didactique que nous avons créé offre de nombreuses opportunités de développement des connaissances métalinguistiques. En effet, lors de discussion sur le futur périphrastique, les locuteurs académiques d'espagnol apportent des éclaircissements sur sa structure grammaticale, alors que les locuteurs d'héritage discutent de son bon usage commun. 
Cela permet dans les deux cas de renforcer la connaissance méta, qu'elle soit applicable au français, à l'espagnol ou même à l'anglais.

En sus du développement des connaissances métalinguistiques, l'anglais et l'espagnol offrent l'avantage d'être des langues apparentées et transparentes qui permettent de mettre en place des stratégies d'intercompréhension encourageant une évolution en mimétisme des processus de translanguaging (Fig. 6b et 6c). D'autres facteurs ont aussi une influence comme un apprentissage académique d'une des deux langues préacquises en tant que seconde langue, des connaissances en linguistique, ou même la connaissance d'une tierce langue romane.

Nous poussons donc les apprenants à comprendre et dépasser les notions normatives de langue pour que leurs expériences d'apprentissage émergent d'un milieu où les langues préacquises et les phénomènes translangagiers, ainsi que la traduction, deviennent partie intégrante de leur multicompétence croissante (Fig. 6a, 6b et 6c). Le français y est bien évidemment présent, et s'y développe en conjonction et en chevauchement (overlap) avec l'espagnol, l'anglais et le spanglish et par le biais de tous les chemins de communication, d'échanges et de transfert existant entre toutes ces zones linguistiques. Ces chemins de réflexions et de communications translangagiers sont ce que nous retrouvons dans ces cours de langue avec approche intercompréhensive utilisant Juntos ainsi que dans un cours intégralement dédié à l'intercompréhension que nous décrivons dans la section suivante.

\section{Intercomprehension of the Romance langages and English}

In an effort to offer other opportunities for students to expand their multilingual repertoire, we have developed a separate course called "Intercomprehension of the Romance Languages." 3 This course is not a language course per se; learners who speak English and a Romance language acquire a reading proficiency of five Romance languages at the same time: Portuguese, Catalan, Spanish, Italian, and French. ${ }^{4}$ In this course, translingual acquisition processes intervene in three manners:

\footnotetext{
${ }^{3}$ Donato and Oliva piloted the course for the first in the United States in spring 2014 at CSULB. Oliva offered the course as a short-term study-abroad program on the campus of the University of Corsica for CSULB students during the summers of 2015, 2016, and 2017. In spring 2018, Donato offered the course at CSULB and Oliva at St. Lawrence University in Canton, NY.

${ }^{4}$ These languages are listed here in the order in which they are presented in EuRom5 (Bonvino, Caddéo, Vilaginés Serra, \& Pippa, 2011). This order (from west to east along the geographical continuum) reflects the original linguistic proximity of the languages, with the exception of French, which is placed last, based on its grammatical, lexical, and syntactical particularities.
} 
- through the use of the pre-acquired repertoire to understand unknown written content

- through the use and the expansion of connective pathways among languages

- through multilingual communication patterns that serve as a delivery mode

\section{Interlexicalité et intergrammaticalité}

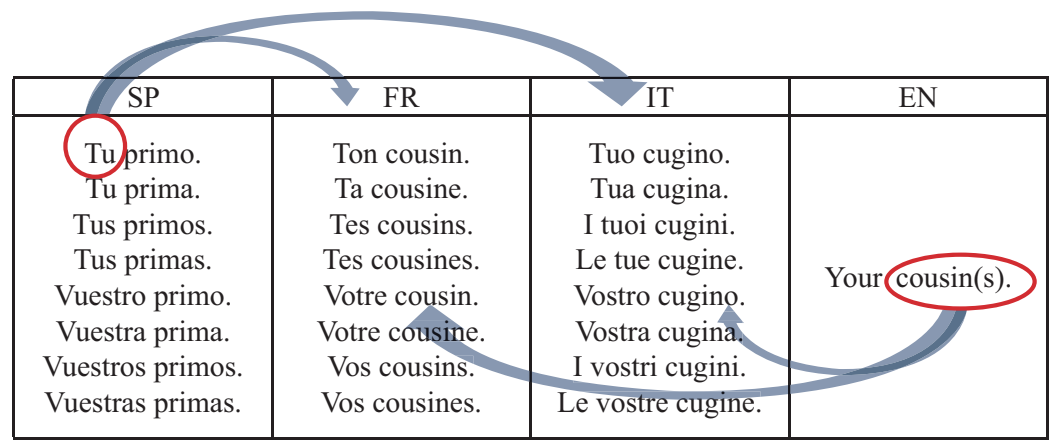

\section{Figure 7}

Interlexicality and intergrammaticality

The course uses the EuRom5 platform (Bonvino et al., 2011), which offers a unique approach to language learning and language teaching via intercomprehensive strategies where learners transcend traditional language learning skills in reading and, to some extent, listening (Caddéo \& Jamet, 2013). Learners start from their repertoire and work on language proximity with the other languages (Fig. 7) by functioning through translingual translation processes, both orally and in writing, whether in the classroom or at home. This allows for an accelerated acquisition of reading competencies at an advanced level in all languages as described by Bonvino et al. (2011) "l'apprenant] arrive très rapidement $[40$ heures (EuRom5, n.d.)] a un niveau de compétences de lecture qui varie entre B1 et B2 du CECR" (p. 69), which is roughly the equivalent of an Intermediate High to Advanced Low on the ACTFL rating scale (ACTFL, 2016):

Dans l'enseignement des langues dit traditionnel (à l'école comme à l' [u]niversité), les langues sont conçues comme des unités distinctes à étudier séparément. Les comparaisons se limitent généralement à une L1 vers une L2. EuRom 5 contribue clairement à dépasser cette vision binaire et permet de considérer que dans un monde où la pluralité des langues et des cultures est la norme, 
il est nécessaire d'affronter l'apprentissage et l'enseignement des langues d'un point de vue plurilingue. (Bonvino et al., 2011, p. 68)

In order to illustrate the innovative aspects of this course for students specializing in language and linguistic studies, we share the experience of Francesca Ricciardelli, co-author, and student in the CSULB Italian masters' program, who intends to continue her work in intercomprehension at the doctoral level and who took the intercomprehension course in spring 2018:

Le livre EuRom5 contient vingt lectures par langue, réparties sur 3 niveaux avec 7 articles [à chaque niveau]. Chaque semaine nous [les étudiants] devons traduire deux textes de langues différentes, mais du même niveau. ... Nous choisissons la langue cible pour la traduction, y compris l'anglais. Dans un premier temps, les traductions étaient difficiles, mais, de semaine en semaine, nous affinons notre capacité à traduire des textes de difficulté croissante dans les langues que nous ne connaissons pas ou peu au début de l'apprentissage. Le succès de nos traductions dans les langues [qui étaient] inconnues avant le cours est rendu possible grâce aux instruments didactiques offerts par le livre. ... L'aide du livre sous forme de tableaux de grammaire comparative et d'enregistrements oraux des lectures, accessibles sur le site en ligne, a été fondamentale pour accomplir des traductions avec nos propres moyens multilingues développés pendant le cours. (Francesca Ricciardelli, personal interview, May 3, 2018)

\section{Crianca nasceu em ambulância a caminho de Viseu}

Uma criança do sexo masculino nasceu hoje, cerca das $12 \mathrm{~h} 00$, numa ambulância dos bombeiros de Resende, a cerca de 12 quilómetros de Viseu, quando se dirigia para o Hospital São Teotónio.

\begin{tabular}{|c|c|}
\hline PT & bombeiros \\
\hline ES & bomberos \\
\hline CA & bombers \\
\hline IT & pompieri \\
\hline F & pompiers \\
\hline
\end{tabular}

\begin{tabular}{|c|c|}
\hline PT & cerca das \\
\hline ES & cerca de las \\
\hline CA & cap a les \\
\hline IT & circa alle \\
\hline F & vers \\
\hline
\end{tabular}

$$
\text { quando } \frac{\text { [ela] }}{\mathrm{S}} \quad \frac{\text { se dirigia }}{\mathrm{V}}
$$

\section{Figure 8}

EuRom5 (Bonvino et al., 2011): Title and first sentence of text P1A with selected examples of lexical and grammatical elicitations

In other contexts, the course can be conducted in a single language, the one that is shared by all students. In our classes, the discussion of a prepared text 
(Fig. 8) in one of the five Romance languages is carried out collaboratively in multiple languages by all students, who often translanguage as they speak and write, drawing from their various linguistic repertoires. The repertoire always includes English as a common resource when the need arises, but the use of less-known languages is seen as a facilitating factor. In a very different context, Portolés and Martí's research (2017) on English as an L3 in the case of speakers of Catalan and Spanish invites us to think of that flexible shift in languages in the classroom as one that
does not merely imply a random shift of code choice, due to lack of linguistic knowledge, but the complete use of one's multilingual repertoire as an interre- lated system of resources, acting ... as a facilitator in the understanding and processing of the target language[s]. (p. 69)

\begin{abstract}
Pour évoquer le fonctionnement pratique [de manière générale], notre professeur nous parle en français, italien et espagnol, [et anglais]. Les étudiants répondent dans ces mêmes langues et des commentaires s'ajoutent aussi de la part de deux étudiants qui parlent le portugais et une étudiante qui connaît le catalan. ... [C] ]ette approche ... est indispensable pour permettre aux étudiants d'être complètement absorbés par plus d'une langue et, surtout, pour obtenir la confiance nécessaire pour parler une langue ... peu connue. (Ricciardelli, personal interview, May 3, 2018)
\end{abstract}

The teacher's approach plays a pivotal role. This uninhibited use of multiple languages initiates a de-dramatization process reinforced by no hierarchical treatment of the five languages (Fig. 8), leading to an environment that offers no linguistic barriers to communicative translanguaging. The course not only encourages students to rethink their communication habits in new languages, but also to redefine what it means "to be fluent in" or "to know" a language. Any new linguistic skill, which may otherwise be described as "passive" or "partial" because it may essentially be of a receptive nature, is valorized here as an additional tool supplementing the learner's thriving repertoire.

Dès la première leçon, le concept des compétences partielles a été introduit, discuté et analysé. Cela nous a immédiatement aidés à comprendre qu'il n'est pas nécessaire d'être capable de parler, lire, écouter et écrire à la perfection pour comprendre et même communiquer dans une autre langue. De plus, les [autres] élèves ont constaté que, au contraire de ce qui fait le fondement des cours de langues traditionnels aujourd'hui, embrasser ses langues déjà connues pour en comprendre d'autres de la même famille linguistique peut devenir une stratégie très utile et, de fait, productive. (Ricciardelli, personal interview, May 3, 2018)

In similar fashion, in Oliva's Intercomprehension course taught at St. Lawrence University in spring 2018, the transcriptions (Fig. 9) exemplify how the use of the full repertoire allows for linguistic expansion through the intercomprehension techniques of bridging and relaying (Castagne, 2007), based on similarities and discrepancies. This work relies on the validation and elicitation of 
pre-acquired knowledge and skills not solely the "content" learned in class (Cenoz, 2017; Cenoz and Gorter, 2015). In the following interview transcripts, both students are English-speaking, with minors in French (student \#1) and Spanish (student \#2). Students were asked to compile data from texts in the five EuRom languages plus English, to argue the correlations and divergences between "there is/there are" and their equivalent forms in other languages.

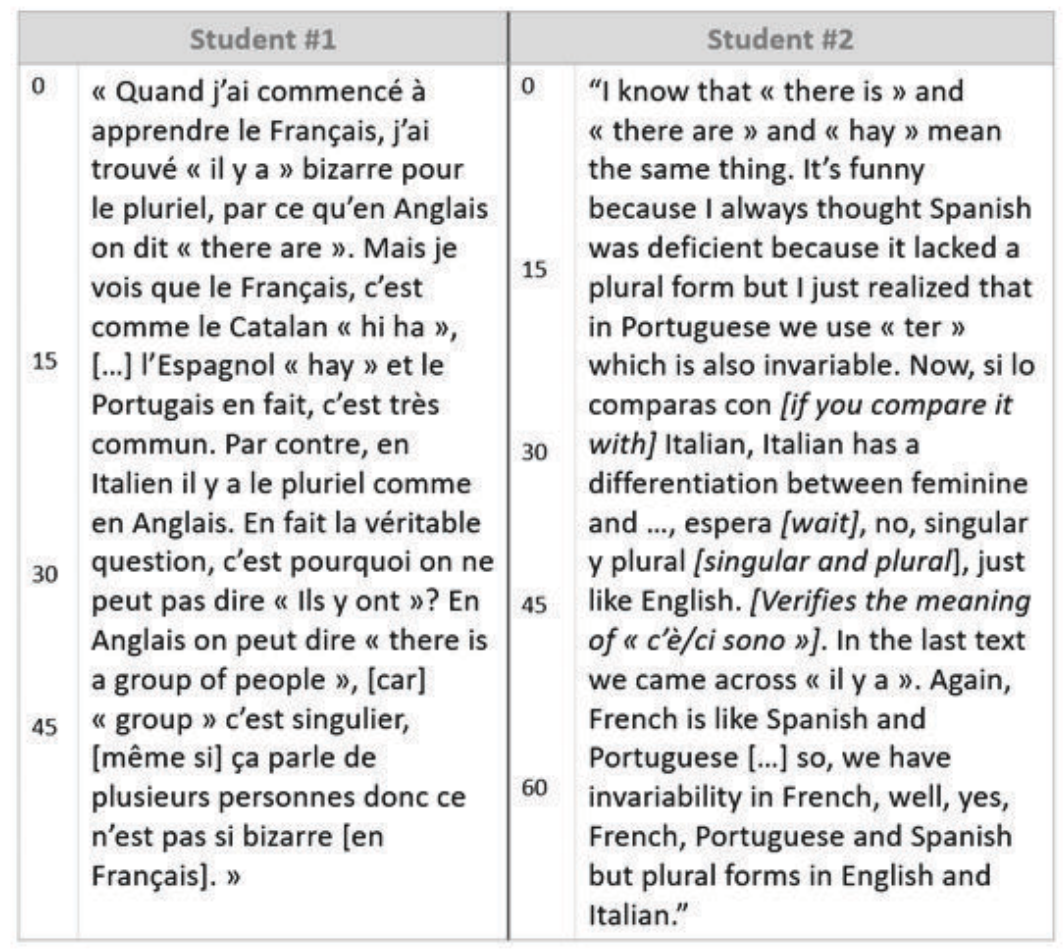

Figure 9

Oliva's intercomprehension course at St. Lawrence University, Spring 2018. Interview question: "Discuss the correlation and differences between "there is/there are" and their equivalent forms in the other languages (Portuguese, Spanish, Catalan, French and Italian).

The students were not provided with any ancillary grammatical tools. They solely enacted their multilingual language repertoire and intercomprehensive metalinguistic skills (acquired in the courses) in the effort to comprehend the Romance language content presented to them. As a result, students explicitly develop their own understanding of interlingual grammar by weaving their 
multilingual competencies together with the metalinguistic knowledge that they are elaborating as their work with multiple Romance languages.

En outre, chaque semaine nous devions lire des articles académiques pour stimuler des discussions en classe et sur la plateforme en ligne [afin que l'] on puisse discuter avant, pendant et après la leçon et réfléchir ensemble sur ce que nous avions découvert sur des sujets pertinents comme la métacognition des langues, l'apprentissage des langues apparentées, les compétences partielles, les intelligences multiples, et la pédagogie, entre autres. (Ricciardelli, personal interview, May 3, 2018)

In order to assess the progress of the students toward the goal of Intercomprehension, Dr. Cortés Velásquez, an intercomprehension specialist at the University of Roma Tre who visited the course at CSULB, hosted a focus group at the end of the course. The transcripts of the recorded focus group sessions were compiled by Ricciardelli and reflect students' perspectives on the course and its outcomes.

One of our heritage speakers of Spanish declared: "Io voglio continuare il mio studio di francese e italiano, ... e ora voglio migliorare il mio livello di spagnolo accademico", 5 demonstrating a heightened interest in expanding her knowledge of those languages that were already present in her linguistic background. The response of this student truly underscores the advantage of multilingual learning, particularly for heritage speakers. In this case, the student, a heritage speaker of Spanish, wishes to both deepen her knowledge of French and Italian but also acquire an academic level of Spanish, which resembles that of the other languages she is learning. This student, like many others, has recognized that there is an academic register of her heritage language, and now sees multiple possibilities for the application of that heritage language, which in all likelihood has never been studied formally:

La fluidité de ce type de translanguaging en classe a engendré une communication efficace et productive qui a renfoncé la motivation des étudiants de multiples manières, notamment [avec la] perspective d'une application pratique dans la vie quotidienne. (Ricciardelli, personal interview, May 3, 2018)

One of our students, who has worked as a teaching associate in both French and Italian, noted that this class helped her understand the importance of bridge languages in Romance language teaching. She became aware of the extent to which heritage Spanish speakers could rely on their Spanish as they bridge to learning French or Italian:

\footnotetext{
${ }^{5}$ Our translation: "I want to keep on studying French and Italian ... and I now want to ameliorate my level of academic Spanish."
} 
[P] arce que je suis professeur, j' ai [a]pris des choses, ... et je [les] ai appliquées avec les étudiants, parce qu'avant ... de prendre ce cours j'avais peur de parler espagnol et je pense que j'ai créé ce mur moi-même, je sais pas pourquoi! Mais ce cours m'a donné la capacité de comprendre les nuances de la langue et moi je les utilise avec mes étudiants pour [leur bénéfice] et je pense que ça c'était le mieux pour eux, le mieux pour moi et le mieux pour tout le monde. (Ricciardelli, personal interview, May 3, 2018)

One of the students affirms that this class helped him gain more confidence in languages he would like to use in a professional context. He had begun learning Catalan shortly before taking the class on Intercomprehension, which he found useful for his career goals: "Vorrei fare il traduttore ... e vorrei farl[o] in altre lingue romanze, per esempio, la settimana scorsa ho fatto dall'italiano al catalano anche se non parlo catalano bene ma riesco a fare la traduzione ... tradurlo perché la lingua scritta la conosco meglio."6

The course never imposed a lingua franca but rather fostered principles of linguistic openness, inclusion, and acceptance that offer opportunities for personal growth. Such multilingual practices can be found in both EuRom5 and the Juntos series.

\section{Conclusion}

As a coda to this article describing the courses we have created and the reaction of our students, it behooves us to mention a two-part series in the Los Angeles Times devoted to the individual language repertoires of residents of the greater Los Angeles region (Bermudez, 2018a). The author of these articles was prompted to write the series when confronted by a woman in a park who reprimanded her for speaking to her daughter in Spanish. The woman told Ms. Bermudez that she was certainly confusing her child, to which she answered that her daughter spoke three languages. The incident sparked a meditation by Bermudez on the great divide between monolingual America and the bi-, tri- and any-number-of lingual adults, and increasingly, of children in Southern California. These two groups are as far apart as they could possibly be. For the second article in the series, Bermudez invited Angelenos (residents of Los Angeles) to share their own linguistic trajectories (Bermudez, 2018 b). The outpouring of emotion over linguistic gain and loss in immigrant families that either encouraged or discouraged bilingual practices in the home, the desire to recuperate or expand one's linguistic repertoire as a quintessential element of one's identity, and the celebration of the richness of life in

\footnotetext{
${ }^{6}$ Our translation: "I would like to become a translator ... and I would like to translate from other Romance languages, for example, last week I've done it from Catalan to Italian even if I do not speak much Catalan, yet I can do the translation ... translate it because I know the written language well."
} 
multiple languages nearly jumped off the page. The unmitigated desire for language and the desire to ensure that future generations experience as many languages as possible, and, above all, be free to live their entire linguistic repertoire, resonated among all of those cited with overwhelming consensus. Although the multilingual subjects who offered their stories in response to Bermudez' call are lacking in the terminology of our profession, they all sought to better understand how their identities were fulsomely realized through language, and diminished when one of their languages was denied to them through shame and the need to assimilate. These people, all of them, speak the hopes, dreams, and concerns of our students who flourish in classroom settings that engage their identity in all of their languages and the learning potential that they possess.

\section{References}

American Council on the Teaching of Foreign Languages ACTFL. (2016). Assigning CEFR ratings to ACTFL assessments. www.actfl.org/sites/default/files/reports/Assigning CEFR_Ratings_To_ACTFL_Assessments.pdf

American Council on the Teaching of Foreign Languages (ACTFL). (n.d.). French: Speaking, Writing, Listening, Reading. www.actfl.org/publications/guidelines-and-manuals/actfl-proficiency-guidelines-2012/french

Babault, S. (2015). L'éducation bilingue: enjeux de politique linguistique, appropriation par les acteurs sociaux et construction de compétences chez les apprenants. Lille, France: Presses universitaires de Lille (PUL).

Bailey, B. (2007). Heteroglossia and boundaries. In M. Heller (Ed.), Bilingualism: A social approach, (pp. 257-276). Basingstoke, UK: Palgrave.

Bermudez, E. (2018a, June 16). I am raising my daughter to speak three languages: A stranger demanded I 'speak English' to her. Los Angeles Times. www.latimes.com/ local/california/la-me-speak-english-20180616-story.html

Bermudez, E. (2018b, July 8). How has language shaped your world? Los Angeles Times. Retrieved from: www.latimes.com/projects/la-me-language-you-speak-responses

Bonvino, E., Caddéo, S., Vilaginés Serra, E., \& Pippa, S. (2011). EuRom5: Ler e compreender 5 línguas românicas/Leer y entender 5 lenguas románicas/Llegir i entendre 5 llengües romàniques/Leggere e capire 5 lingue romanze/Lire et comprendre 5 langues romanes. Milan, Italy: Hoepli.

Burck, C. (2005). Multilingual living: explorations of language and subjectivity. London: Macmillan.

Caddéo, S. et Jamet, M.C. (2013). L'intercomprehension: une autre approche pour l'enseignement des langues. Paris: Hachette.

California State University. (2019). Institutional research and analyses, Statistical reports 2018-2019: CSU Enrollment by Ethnic Group and Student Level, Fall 2018 
(Table 3: Fall 2018 Profile CSU Enrollment by Ethnic Group and Student Level, Number and Ratio to Totals, Systemwide). Long Beach, CA: California State University. calstate.edu/as/stat_reports/2018-2019/feth03.htm

Canagarajah, S. (2011). Codemeshing in academic writing: Identifying teachable strategies of translanguaging. Modern Language Journal, 95, 401-417. doi.org/10.1111/j.1540-4781.2011.01207.x

Carvalho, A.M. \& Child, M. (2018). Expanding the multilingual repertoire: Teaching cognate languages to heritage Spanish speakers. In K. Patowsky (Ed.), The Routledge handbook of Spanish as a heritage language (pp. 420-432). New York: Routledge.

Carvalho, A.M., Freire, J.L., \& da Silva, J.B. (2010). Teaching Portuguese to Spanish speakers: A case for trilingualism. Hispania, 93, 70-75.

Castagne, E. (2007). Transparence lexicales entre langues voisines. Dans E. Castagne (Dir.), Les enjeux de l'intercompréhension/The stakes of intercomprehension (pp. 155-166). Reims, France: Èditions et Presses universitaires de Reims (ÉPURE).

Cenoz, J. (2017). Translanguaging pedagogies and English as a lingua franca. Language Teaching, 52, 71-85. doi.org/10.1017/S0261444817000246

Cenoz, J., \& Gorter, D. (2015). Towards a holistic approach in the study of multilingual education. In J. Cenoz \& D. Gorter (Eds.), Multilingual education: Between language learning and translanguaging (pp. 1-15). Cambridge: Cambridge University Press.

Cook, V.J. (1992). Evidence of multi-competence. Language Learning, 44, pp. 557591. doi.org/10.1111/j.1467-1770.1992.tb01044.x

Creese, A., \& Blackledge, A. (2010). Translanguaging in the bilingual classroom: A pedagogy for learning and teaching? Modern Language Journal, 94, 103-115. doi.org/10.1111/j.1540-4781.2009.00986.x

Cummins, J. (2005). A proposal for action: Strategies for recognizing heritage language competence as a learning resource within the mainstream classroom. Modern Language Journal, 89, 585-592.

De Pietro, J.F. et Rispail, M. (2014). L'enseignement du français à l'heure du plurilinguisme: vers une didactique conceptualisée. Namur, Belgique: Les Presses universitaires de Namur (PUN).

Donato, C. \& Oliva, C.J. (2014). The ties that bind: Italian for Spanish speakers in intercomprehension. In R. Dolci (Ed.), Intercomprehension and multilingualism: Teaching Italian to Romance languages speakers (pp. 61-78). New York: Calandra Institute Transactions.

Donato, C. \& Oliva, C.J. (2016). The future is multilingual: French, Italian, and Portuguese for Spanish speakers. ADFL Bulletin, 44, 112-127. 
Donato, C., Oliva, C.J., Zappador-Guerra, D., \& Romero, M. (2014). Juntos: Italian for speakers of English and Spanish - Student guide. Long Beach, California: George L. Graziadio Center for Italian Studies. csu.redshelf.com/book/53536/juntos-italian-forspeakers-of-english-and-spanish-53536-9780990728801-clorinda-donato-cedric-joseph-artoliva-manuel-romero-daniela-zappador-guerra

Escudé, P. et Janin, P. (2010). Le point sur l'intercompréhension, clé du plurilinguisme. Paris: CLE International.

EuRom5. (n.d.). L'histoire d'EuRom4. www.eurom5.com/p/chisiamo-fr/lastoria

García, O. (2007). Foreword. In S. Makoni \& A. Pennycook (Ed.), Disinventing and reconstituting languages (pp. xi-xv). Clevedon, UK: Multilingual Matters.

García, O. (2009). Bilingual education in the 21st century: A global perspective. Oxford: Basil/Blackwell.

García, O. (2011). From language garden to sustainable languaging: Bilingual education in a global world. Perspectives: A publication of the National Association for Bilingual Education, Sept/Oct, 5-9. ofeliagarciadotorg.files.wordpress.com/2011/02/ 2012nabenewsletter_34n1_nov2011_dec2011.pdf

Gramling, D., \& Warner, C. (2015). Introduction to the issue: In memory of Richard Ruiz (1948-2015). Critical Multilingualism Studies, 3, 1-6. cms.arizona.edu/ojs3/ multilingual/article/download/72/110

Grosjean, F. (2010). Bilingual: Life and reality. Cambridge, MA: Harvard University Press.

Jaffe, A. (1999). Ideologies in action: Language politics on Corsica. Berlin: Mouton de Gruyter.

Kramsch, C. (2009). The multilingual subject: What foreign language learners say about their experience and why it matters. Oxford, UK: Oxford University Press.

Kramsch, C., \& Zhang, L. (2018). The multilingual instructor: What foreign language teachers say about their experience and why it matters. Oxford, UK: Oxford University Press.

Lemke, J.L. (2002). Travels in hypermodality. Visual Communication, 1, 299-325.

Lin, A.M.Y., \& Martin, P. (2005). Decolonisation, globalisation: Language-ineducation policy and practice. Clevedon, UK: Multilingual Matters.

Makoni, S., \& Mashiri, P. (2007). Critical historiography: Does language planning in Africa need a construct of language as part of its theoretical apparatus? In S. Makoni \& A. Pennycook (Eds.), Disinventing and reconstituting languages (pp. 62-89). Clevedon, UK: Multilingual Matters.

Oliva, C.J. (2011, May). The potential place of "minority” languages in language education through 'intercomprehension'. Poster session presented at the Journeée des doctorants [May, June], Pasquale Paoli University of Corsica, Corte, Corsica, France. 
Oliva, C.J. (2014-2016). [Comprehensive survey of French and Italian for speakers of English and Spanish courses and traditional courses at California State University, Long Beach]. 506 participants. Unpublished raw data.

Oliva, C.J. (2016, Feb.) Les enjeux pour l'apprentissage du français au travers de l'intercompréhension. Paper presented at the 4th International FLE Colloquium, University of Puerto Rico, Río Piedras campus, San Juan, PR.

Oliva, C.J. et Donato, C. (2015). Nouveau public, nouvelle stratégie: Français pour locuteurs anglo-hispanophones. Crisolenguas [numéro thématique: Didactique du FLE à réinventer: mondialisation, immigration et référents culturels en coportage], $3,58-71$.

Oliva, C.J., Donato, C., Gomez, A., \& Romero, M. (2015) Juntos: French for speakers of English and Spanish-Student guide. Long Beach, California: George L. Graziadio Center for Italian Studies.

csu.redshelf.com/book/257997/juntosfrenchforspeakersofenglishandspanish257997nonecedric josepholivaclorindadonatoalangomezlarrivaandmanuelromero

Pabon, T., \& Rodriguez, M. (1967). I like it like that [Recorded by Cardi B, featuring Bad Bunny and J Balvin]. On Invasion of Privacy [Record album]. New York: Atlantic Records. (April 2018).

Perez, C. (2015, June 29). US has more Spanish speakers than Spain. New York Post. nypost.com/2015/06/29/us-has-more-spanish-speakers-than-spain

Portolés, L., \& Martí, O. (2017). Translanguaging as a teaching resource in early language learning of English as an additional language (EAL). Bellaterra Journal of teaching \& Learning Language \& Literature, 10, pp. 61-77. revistes.uab.cat/jt3/ article/view/v10-n1-portoles-marti

Pratt, M.L. (2011, April). Globalization as linguistic force field [Plenary keynote lecture]. Paper presented at 64th Annual Kentucky Foreign Language Conference, University of Kentucky, Louisville.

Ricciardelli, F. (2018) [Transcripts from recordings of focus group conducted by Dr. Cortés Velásquez at California State University, Long Beach (CSULB), Spring 2018]. Unpublished raw data.

Ruben, N. (2014, April 7). California Hispanic milestone. CNN. edition.cnn.com/2014/04/ 07/opinion/navarrette-california-hispanics/index.html

Schmidt, R.W. (2010). Attention, awareness, and individual differences in language learning. In W.N. Chan et al. (Eds.), Proceedings of the Fourth CLS International Conference (CLaSIC) 2010 Individual characteristics and subjective variables in language learning (pp. 721-737). Singapore: National University of Singapore, Centre for Language Studies. nflrc.hawaii.edu/PDFs/SCHMIDT\%20Attention, \%20awareness, \%20and\%20individual\%20differences.pdf

Seidlhofer, B. (2003). A concept of international English and related issues: From 'real' English to 'realistic' English. Strasbourg, France: Council of Europe. citeseerx.ist.psu.edu/viewdoc/download?doi=10.1.1.475.6022\&rep=rep1\&type=pdf 
Simon, S. (2012). Cities in translation: Intersections of language and memory. London: Routledge.

Stevens, P. (1990). Teaching French to Spanish speakers: Some typical patterns of error. Papers and Studies in Contrastive Linguistics, 26, 29-38. files.eric.ed.gov/fulltext/ ED341244.pdf

Williams, C. (1994). Arfarniad o ddulliau dysgu ac addysgu yng nghyd-destun addysg uwchradd ddwyieithog [An evaluation of teaching and learning methods in the context of bilingual secondary education] (Unpublished doctoral dissertation). Bangor, UK: University of Wales. 